\title{
EcoFlex: A Multifunctional MoClo Kit for E. coli Synthetic Biology
}

\author{
Simon J. Moore, ${ }^{\dagger, \S}$ Hung-En Lai, ${ }^{\dagger, \S}$ Richard J. R. Kelwick, ${ }^{\dagger, \S}$ Soo Mei Chee, ${ }^{\S}$ David J. Bell, ${ }^{\S}$ \\ Karen Marie Polizzi, ${ }^{\dagger, \ddagger}$ and Paul S. Freemont ${ }^{*}, \dagger, \S$
}

${ }^{\dagger}$ Centre for Synthetic Biology and Innovation, Imperial College London, South Kensington Campus, Exhibition Road, London, SW7 2AZ, U.K.

${ }^{\ddagger}$ Department of Life Sciences, Imperial College London, South Kensington Campus, Exhibition Road, London, SW7 2AZ, U.K.

${ }^{\S}$ Department of Medicine, Imperial College London, South Kensington Campus, Exhibition Road, London, SW7 2AZ, U.K.

\section{Supporting Information}

ABSTRACT: Golden Gate cloning is a prominent DNA assembly tool in synthetic biology for the assembly of plasmid constructs often used in combinatorial pathway optimization, with a number of assembly kits developed specifically for yeast and plant-based expression. However, its use for synthetic biology in commonly used bacterial systems such as Escherichia coli has surprisingly been overlooked. Here, we introduce EcoFlex a simplified modular package of DNA parts for a variety of applications in E. coli, cell-free protein synthesis, protein purification and hierarchical assembly of transcription units based on the MoClo assembly standard. The kit features a library of constitutive promoters, T7 expression, RBS strength variants, synthetic terminators, protein purification tags and fluorescence proteins. We validate EcoFlex by assembling a 68-part containing (20 genes) plasmid (31 kb), characterize in vivo and in vitro library parts, and perform combinatorial pathway assembly, using pooled libraries of either

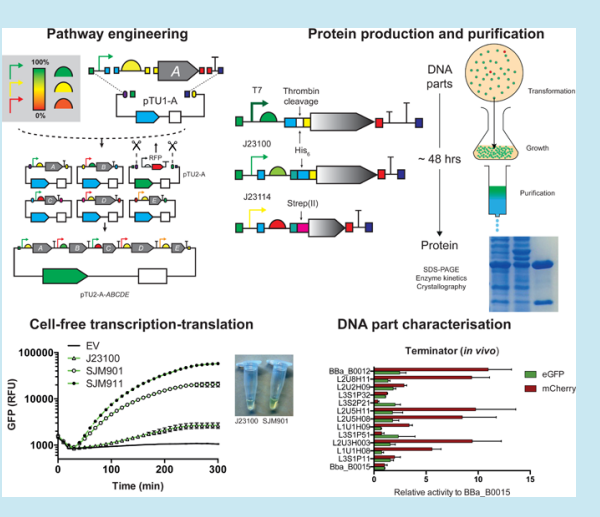
fluorescent proteins or the biosynthetic genes for the antimicrobial pigment violacein as a proof-of-concept. To minimize pathway screening, we also introduce a secondary module design site to simplify MoClo pathway optimization. In summary, EcoFlex provides a standardized and multifunctional kit for a variety of applications in E. coli synthetic biology.

KEYWORDS: synthetic biology, Golden Gate, Escherichia coli, recombinant protein production, cell-free protein synthesis, violacein

A prime target for synthetic biology is the production of natural and commodity chemical products such as drug precursors, fine and specialized chemicals and biofuels using renewable feedstocks. ${ }^{1}$ This is driven by the need to transition away from a current reliance on petroleum-derived chemical products and production processes. A more sustainable and environmentally friendly solution to this problem is in the application of synthetic biology to engineer enzyme biosynthetic pathways primarily within microbial cells to produce such chemical products. Essential to the synthetic biology workflow is the use of rapid, efficient and hierarchical DNA assembly techniques and a number of methods have been proposed. ${ }^{2}$ One such method, Golden Gate cloning, is a modular, combinatorial technique used for a wide spectrum of DNA assembly applications, from the construction of simple transcription units (TUs) to diverse biochemical pathway libraries originally developed by Marillonnet and colleagues. ${ }^{3-5}$

Golden Gate cloning utilizes the non-palindromic Type IIS restriction endonuclease BsaI, which cuts outside of its recognition sequence (GGTCTCN ${ }^{\downarrow} \mathrm{NNNN}$ ), producing a four base pair overhang. This overhang, also known as a fusion site, can be designed to provide directional DNA part assembly. BsaI sites are placed at both the $5^{\prime}$ and $3^{\prime}$ ends of a DNA part in inverse orientation, allowing the restriction site to be removed before assembly by the T4 DNA ligase. DNA parts are held within "entry vectors" and can include promoters, protein tags, ribosome-binding sites (RBS), open reading frames (ORF), protein domains, TALEN repeats, ${ }^{6}$ fusion linkers and terminators. A combined one-pot reaction of "entry vectors" is then digested and ligated into "destination vectors" using BsaI and T4 DNA ligase. Golden Gate assembly has been demonstrated to assemble up to 10 DNA fragments with greater than $95 \%$ efficiency $^{5}$ and relies solely on the use of the BsaI enzyme. Recently, modifications to the original Golden Gate cloning technique included Golden Braid and MoClo standards, ${ }^{7,8}$ which rely on the use of alternating antibiotic marker plasmids and dual type IIS endonucleases (BsaI/BsmBI or $\mathrm{BsaI} / \mathrm{BpiI}$ ) to rapidly construct hierarchical multigene pathways up to $33 \mathrm{~kb}$ in size. ${ }^{9}$ In summary, several plasmid kits have been developed including MoClo and GoldenBraid for plants, ${ }^{7,10,11}$ Saccharomyces cerevisiae ${ }^{12,13}$ and Schizosaccharomyces pombe. ${ }^{14}$ For a detailed review of assembly strategies utilizing Type IIS endonucleases please see Casini et al. ${ }^{2}$

Special Issue: Synthetic Biology in Europe

Received: January 29, 2016 
Focusing on MoClo, this particular assembly scheme allows flexible design of multiple transcription units into modular pathways with each gene under the control of its own promoter, RBS and terminator.

Until the recent release of the CIDAR MoClo kit, ${ }^{15}$ Golden Gate assembly kits for commonly used bacterial systems such as E. coli have surprisingly been overlooked. CIDAR focuses on an automated workflow for MoClo assembly. However, we wanted to expand this further by modifying and creating a new flexible Golden Gate toolkit specifically for E. coli synthetic biology, based on a custom-designed modular library of standard biological parts for hierarchical DNA assembly and optimization, using the MoClo assembly standard. ${ }^{9}$ Herein, we present EcoFlex, a user-friendly, characterized and customizable Golden Gate kit with parts that can be used for example in combinatorial pathway optimization, cell-free circuit design, recombinant protein purification and, in the future, genome engineering. We have also included a custom-designed library of constitutive $\sigma^{70}$ promoters, inducible expression based on the T7 RNA polymerase, a standardized RBS library and a range of synthetic terminators previously characterized. ${ }^{16}$ The EcoFlex kit also provides options for recombinant protein production and purification, such as N-terminal linkers for hexahistidine and Strep(II)-tag purification, with either constitutive or T7inducible expression as single parts or combined with a $\mathrm{T} 7$ promoter, RBS, $\mathrm{His}_{6}$-tag and thrombin cleavage site. We also provide a plasmid cassette system (and individual parts) for inducible T7 RNA polymerase (RNAP) production and purification (Figure S6). We validate T7 RNAP activity using cell-free protein synthesis (CFPS). We have also made two improvements to the MoClo system, first by simplifying the storage plasmid for open reading frames (ORFs), and second we introduce a secondly site to offer flexibility in pathway design. For example, three TUs can be placed in the secondary module under constant expression, while the primary site can be used to assemble and optimize a specific gene, regulatory cascade or biosynthetic pathway.

In synthetic biology, there is an increasing need to provide a repertoire of biological parts (promoters, RBS, terminators) characterized in the context of the chosen DNA assembly technique. For example, DNA parts can behave differently when placed in a different context (known as "context dependency"), which directly challenges the engineering dogma surrounding synthetic biology. A number of investigators have tried to address this problem through the design of neutral insulator sequences. ${ }^{17-19}$ To understand such effects in our EcoFlex kit, we have validated our library of biological parts both in vivo and in vitro (in cell-free reactions). To demonstrate the broad utility of EcoFlex, we also provide exemplars of rapidly assembling and purifying $\mathrm{His}_{6}$-tagged proteins, rational library design using flow cytometry and the optimization of the violacein biosynthetic pathway using a secondary MoClo module to simplify pathway design and increase the frequency of successful library assemblies.

E. coli is one of the major workhorses in synthetic biology, providing a very well-studied chassis for the manipulation of genetic circuits and implementation of biosynthetic pathways. Using a repertoire of biological parts, modular DNA pathways can be efficiently assembled using a range of techniques such as Golden Gate assembly, ${ }^{3}$ BglBricks, ${ }^{20}$ AQUA, ${ }^{21}$ BASIC $^{22}$ and unique nucleotide sequence (UNS)-guided assembly. ${ }^{23}$ In addition, other cloning methods include PCR and restriction enzyme-based cloning, Gibson assembly ${ }^{24}$ and sequence- ligation independent cloning. ${ }^{25}$ Eventually we desire to assemble a universal cloning system based on the MoClo standard for prokaryotic host expression to allow an ORF or DNA part (e.g., promoter, RBS) to be easily portable between multiple systems. To begin building this foundation, we reveal EcoFlex that is designed to provide users a flexible combinatorial library toolbox that can also be customized for variety of synthetic biology applications in E. coli.

We have built a versatile Golden Gate toolbox based on the MoClo assembly standard ${ }^{9}$ for E. coli synthetic biology, which we make available through AddGene (www.addgene.org/ cloning/moclo/freemont-ecoflex). To design this kit, we focus on providing flexibility and compatibility with a variety of applications such as pathway assembly, cell-free protein synthesis (CFPS) and recombinant protein purification (Figure 1). Promoters, RBS, tags, ORFs and terminator "parts" were assembled and stored in Level 0 "entry vectors" with chloramphenicol resistance gene and pMB1 origin of replication. A unique feature of our assembly kit is the "entry vector" for storing ORFs ( $\mathrm{pBP}-\mathrm{ORF}$ ), whereby to standardize the cloning procedure, each gene is PCR cloned into NdeI and BamHI sites, which are flanked by inverse BsaI sites for Golden Gate assembly. The NdeI (CATATG) site provides the fusion site (in bold) for Golden Gate assembly to the RBS sequence (or N-terminal tag) and the start codon for each gene, so no additional amino acids are added to the encoded protein. Optionally, if NdeI or BamHI sites are located within the gene, a NcoI site is also provided in the vector, while BglII, which produces cohesive ends, can be used in place of BamHI. ORFs can also be cloned with flanking BsaI restriction sites with compatible fusion sites ( $5^{\prime}$ CATA and $3^{\prime}$ TCGA). With NdeI and BamHI cloning, these genes are also suitable for transfer between some of the commercial $\mathrm{pET}$ vectors (e.g., pET-3a, $\mathrm{pET}-14 \mathrm{~b}, \mathrm{pET}-15 \mathrm{~b})$ for the purpose of recombinant protein production. Alternatively, our kit also provides customized Nterminal fusion tags with either constitutive or T7 RNA polymerase-dependent expression. The "entry vectors" pBPlac $Z \alpha$ and $\mathrm{pBP}-\mathrm{ORF}$ are customizable for assembly of new promoter parts or ORFs. Further instructions for user modifications are provided in the SI text.

The first tier of assembly (Level 1) requires BsaI to assemble monocistronic TUs, with each ORF under control of its own unique promoter, RBS and transcription terminator (Figure $1 \mathrm{~A})$. This is ligated into a "destination vector" backbone (pTU1-A, -B, - C, -D) containing a pMB1 origin and $\beta$ lactamase gene. Level 1 fusion sites and assembly options are summarized in Figure 1B. Level 1 TUs contain flanking inverse BsmBI sites, which are subsequently used to direct the MoClo (Level 2) stage of assembly (ABCD). The pTU1-A, -B, -C and -D (Level 1) plasmids allow assembly of 2-4 TUs into the Level 2 "destination vectors" pTU2-a (to accommodate 2 TUs), -b (3 TUs) and pTU2-A, -B, -C and -D (4 TUs). In addition, optional pTU1-D 1 and pTU1-E Level 1 plasmids also allow an additional Golden Gate fusion site for assembly up to 5 TUs in pTU2-A, -B, -C or -D. Level 2 plasmids are available with either lacZ $\alpha$ or RFP negative markers, a selection of origins of replication (pMB1, ColE1 and p15A) and a chloramphenicol resistance marker. For larger pathways, up to 20 TUs (Figure S2) can be assembled into a Level 3 "destination vector" (pTU3-A for 2 pTU2 plasmids; pTU3-B for 4 pTU2 plasmids).

Significantly, we have modified the MoClo system to include a secondary module. This is a unique feature to EcoFlex, 
A
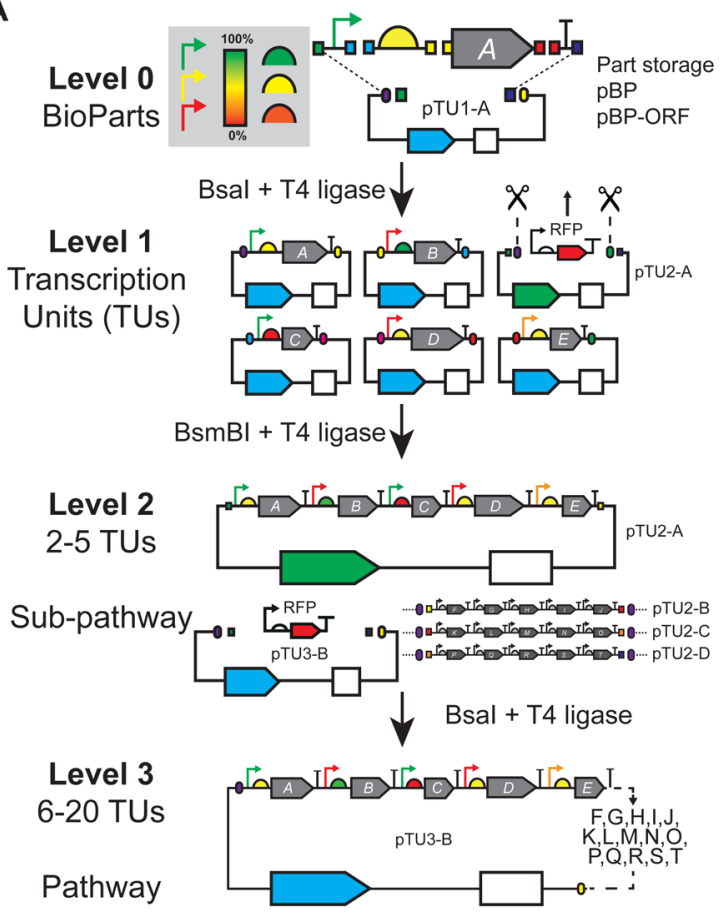
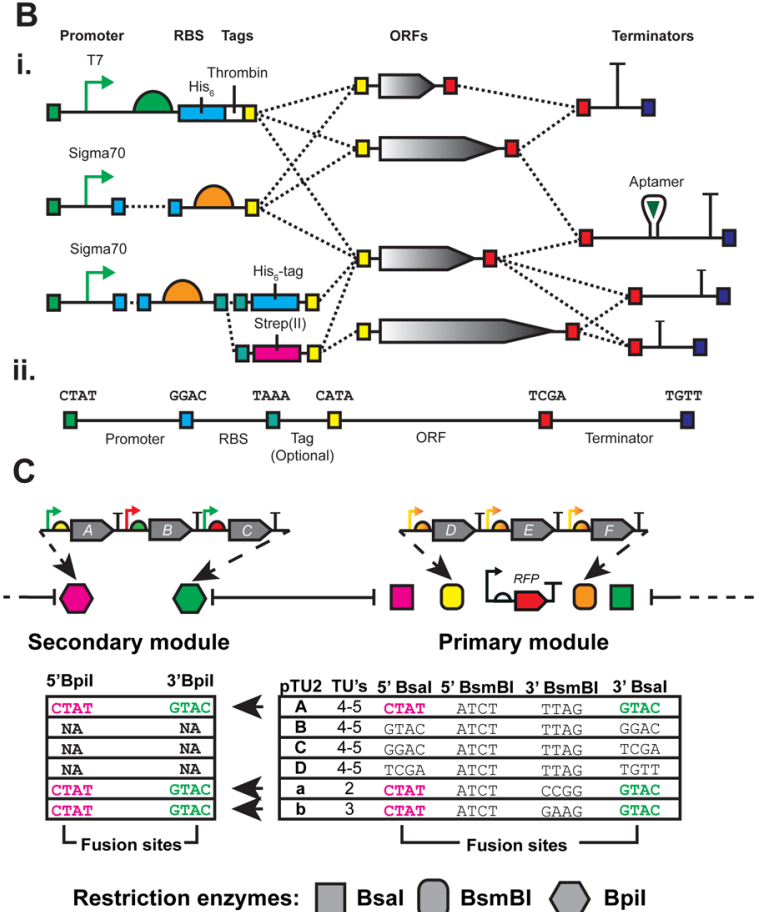

Figure 1. EcoFlex assembly. (A) Level 0 parts are held in pBP (bioparts-promoters, RBS, tags and terminators) and pBP-ORF (open reading frames) plasmids and assembled with BsaI and T4 DNA ligase into pTU1 destination plasmids (A, -B, -C, -D or - $\mathrm{D}_{1}$, -E) forming Level 1 TUs. Genes are cloned with NdeI $\left(5^{\prime}\right)$ and BamHI $\left(3^{\prime}\right)$, with the NdeI site providing an overlap between the start codon and RBS fusion site (CATA). Alternatively, if these sites are located within the gene, custom designed BsaI primers are used. Level 2 modules (2-5 TUs) were assembled with BsmBI and T4 DNA ligase into pTU2 plasmids, which were modified to provide flexibility for assembling 3-5 TUs into a module. In addition, Level 3 pathways (up to $20 \mathrm{TUs}$ ) can be assembled from compatible Level 2 modules into pTU3-A or pTU3-B using BsaI and T4 DNA ligase. (B) Assembly options (i) for purification tags and promoter choice, (ii) fusion site sequences and positions for Level 1 assembly of TUs. (C) Secondary module cloning for optimizing library screening and minimizing pathway variation. Table details compatible fusion sites and assembly options.

whereby separate modules of Level 2 TUs (2-5 TUs) derived from pTU2-a, -b or -A plasmids can be subcloned into a secondary site (pTU2S-A, -a, or -b), using two compatible BpiI sites, which is located upstream of the primary MoClo BsmBI assembly site (Figure 1C). We have introduced this secondary module site as an aid to minimize pathway diversity and increase assembly efficiency during combinatorial optimization. For example, instead of optimizing a six-gene pathway randomly, one module of 2 or 3 TUs can be placed in the secondary module, while the remaining 4 or 3 TUs, respectively, are randomized in the primary module. This is important since the screening technique and transformation efficiency are major limiting factors for library screening. An example of its use is demonstrated with the violacein pathway (see below). Finally, all EcoFlex "destination vectors" mentioned (pTU1, pTU2 and pTU3) also contain a monomeric red fluorescence protein (RFP) gene (except pTU2S-A, -a and -b due to internal BpiI sites in RFP) under a LacI- and CAP-sensitive promoter and strong RBS, sourced from the iGEM Registry of standard biological parts (BBa_J04450). The RFP transcription unit is inserted between flanking BsaI sites (for pTU1 and pTU3) or BsmBI sites (for pTU2) so that negative colony-forming units (CFUs) can be visibly identified (red) after plate growth.

A range of promoter and RBS strength variants were created by randomizing the E. coli $\sigma^{70}$ Anderson promoter collection ${ }^{26}$ and PET RBS Shine-Dalgarno consensus sequences, respectively, upstream of a eGFP (hereafter referred to as GFP) fluorescence reporter with flanking BsaI sites provided for downstream Golden Gate Level 1 assembly (see SI text). We utilized the iGEM Anderson promoters J23100, J23108 and J23114 as internal reference standards. For the RBS library a 6 bp Shine-Dalgarno consensus sequence AGGAGA was randomized to RRRRRR (A/G) to vary ribosome binding strength and a range of low-high strengths are provided. The promoter and RBS strengths in Level 1 context were characterized in the destination vector PTU1-A with a constant J23100/SJM901 promoter (RBS library) or PET RBS (promoter library), GFP and BBa_B0015 terminator. This was performed using E. coli as a chassis with in vivo or in vitro (CFPS) fluorescence measurements. Previously we have demonstrated a link between in vivo and in vitro characterization of promoter and RBS activity. ${ }^{27}$ Herein, we provide further evidence of this relationship by characterizing a wider range of promoter (Figure 2A,E), RBS (Figure 2B) and synthetic terminator parts (Figure 2C,D).

In summary, most of the parts behaved similarly between the two systems. For example, a strong promoter or terminator in vivo was generally strong in vitro. However, there are some exceptions to this general rule. For example, in vivo, the SJM901 promoter variant showed a moderate GFP synthesis rate of 1.2fold in comparison to the J23100 promoter standard. Unexpectedly, in vitro, this rate increased to 7.8-fold (Figure 2A,E,F). While promoter strengths demonstrated a clear link between in vivo and in vitro activity, we observed some differences between RBS and terminator activity, which was dependent on the strength of promoter used. Unlike in vivo, in vitro, the signal-noise ratio for the J23100 promoter was low (Figure S4), thus it is unclear to differentiate between weakstrong RBS variants. This could be improved by using the 


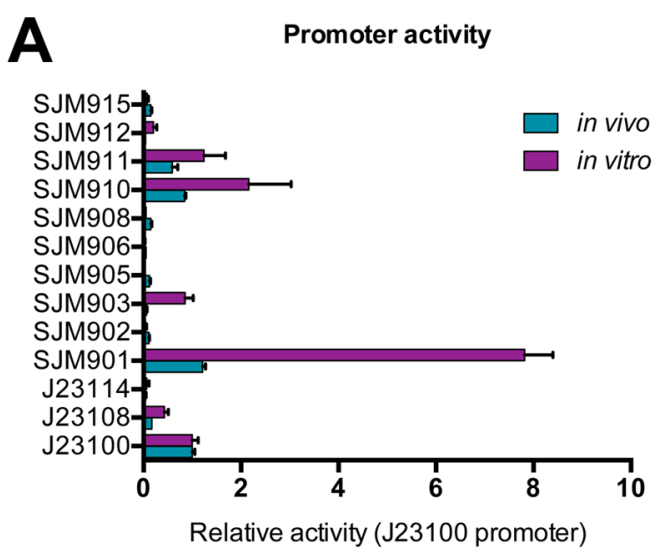

C
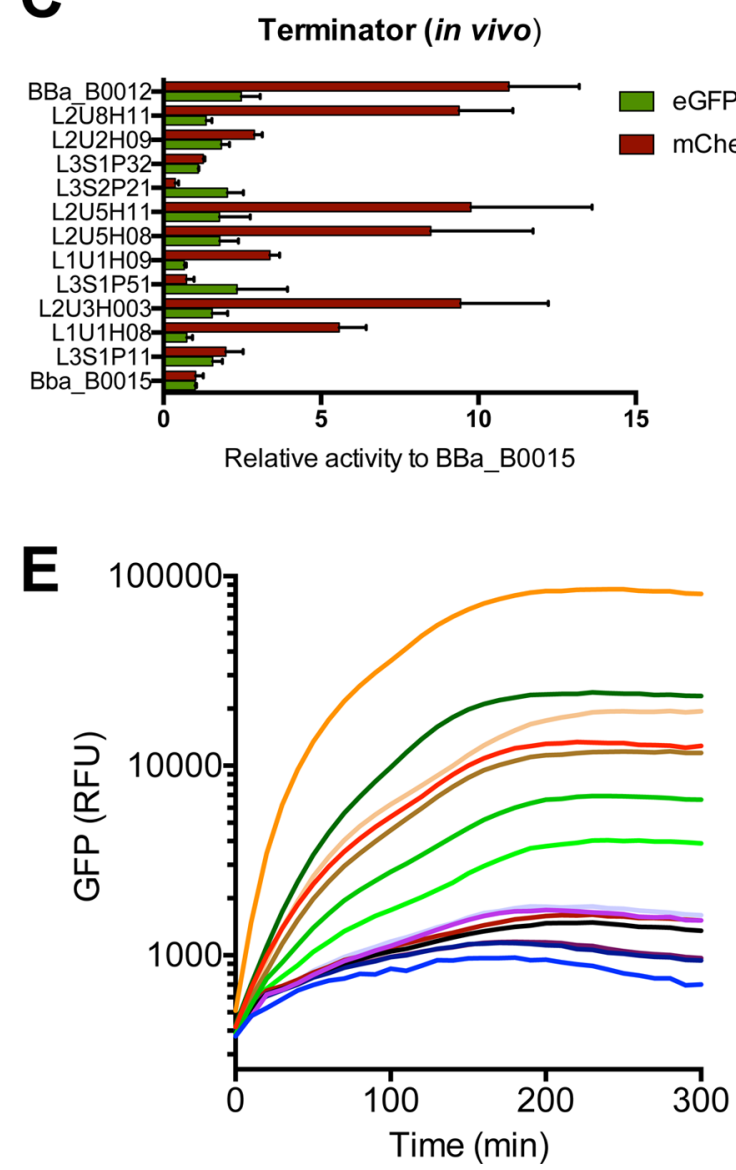

B RBS activity

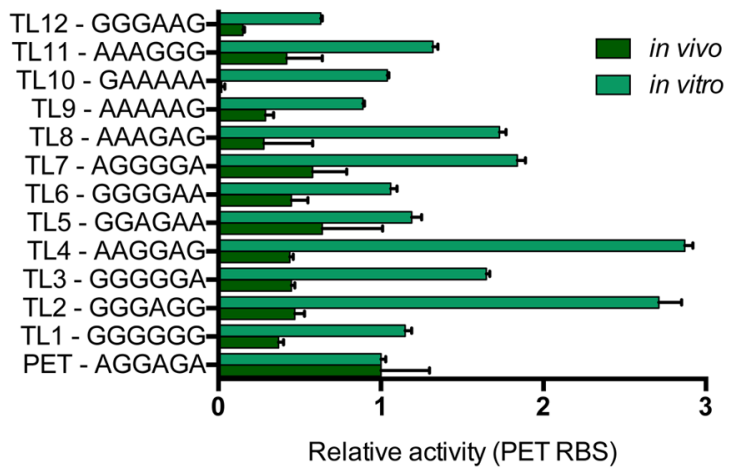

D
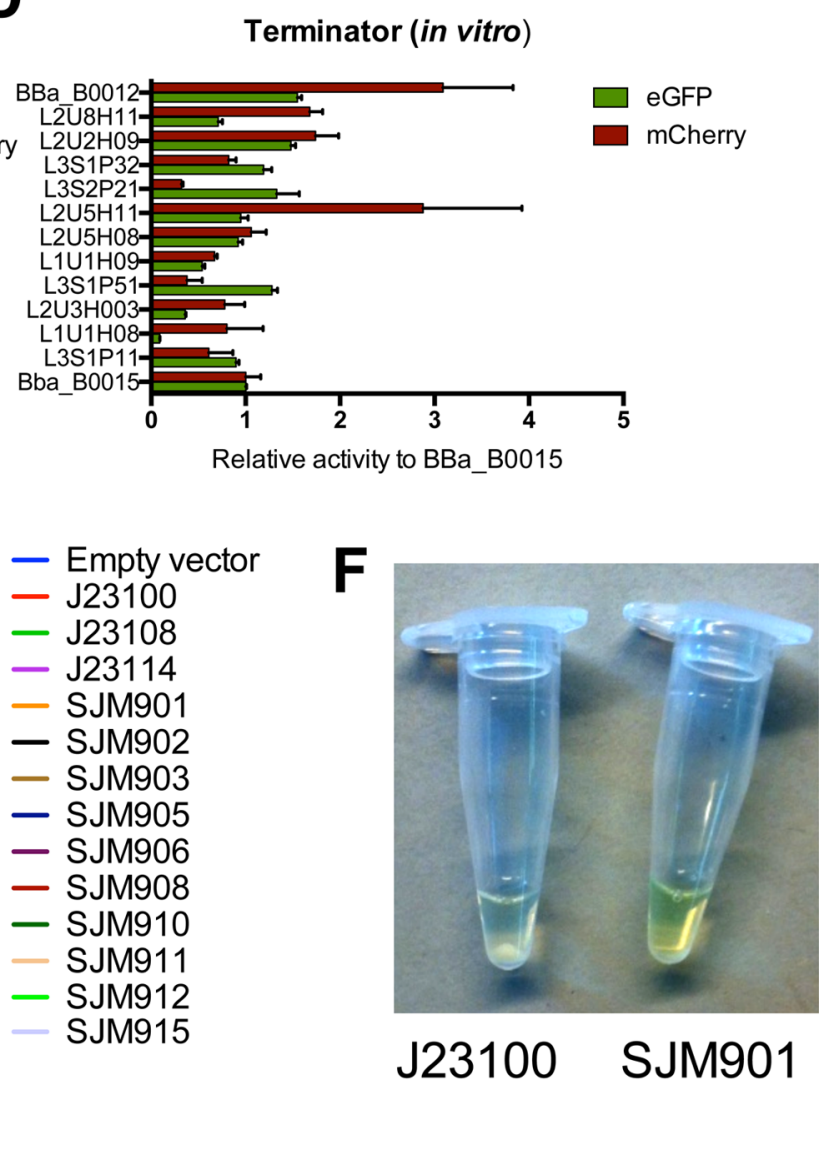

Figure 2. Part characterization in vitro and in vivo. A select range of promoters (A), RBS (B) and terminator variants (C, D) were tested for relative activity in E. coli DH10 $\beta$ and cell-free protein synthesis. The J23100 and PET RBS were used for normalization of promoter and RBS data, respectively. For the terminator assay (Level 2 plasmids), GFP was assembled with a SJM901 promoter, PET RBS and a variable strength terminator. This was followed by a weak J23114 promoter, PET RBS, mCherry and BBa B0015 terminator. Terminator data is represented as normalized relative activity of GFP and mCherry, respectively. (E) The Sigma70 promoter library characterization using cell-free protein synthesis. (F) Visual appearance of GFP production from cell-free extracts with the SJM901 promoter in comparison to J23100.

strong SJM901 promoter (Figure S4). However, with the SJM901 promoter the range of weak-strong RBS variants indicated from in vivo observations was not as clear in vitro, while some variants showed enhanced activity (TL2 and TL4) of almost 3-fold over the PET standard. These findings could suggest that the strong activity of the SJM901 promoter saturates ribosome binding in vitro therefore narrowing the range of observed activities for apparent weak and strong RBS variants.
For terminator characterization, a different assay was designed to quantify terminator efficiency using EcoFlex parts. To do this, GFP was assembled into pTU1-A with the J23100 or SJM901 promoter and PET RBS, followed by a variable strength terminator. In pTU1-B, mCherry was assembled with the weak J23114 promoter, PET RBS and BBa_B0015 terminator. These two TUs were then assembled into pTU2-a as a Level 2 assembly (Figure S3). If the variable terminator allowed read-through transcription, this was 
detected by mCherry fluorescence. In comparison to the original publication describing the synthetic terminator parts ${ }^{16}$ used herein, the simple assay we utilize differs in the use of a strong constitutive promoter instead of the arabinose induction system used previously. ${ }^{16}$ We represent our data as a mCherry and GFP rate that is normalized to a control plasmid containing two strong BBa_B0015 terminators for both GFP and mCherry (Figure 2C,D). Although our data is not a direct comparison to Chen et al., ${ }^{16}$ our in vivo measurements suggest that the activity of the synthetic terminators is reproducible in the EcoFlex context with either the SJM901 (Figure 2C) and J23100 promoters (see SI files). It should also be noted that growth rate of the Level 2 plasmids (chloramphenicol) is considerably slower than for the promoter and RBS characterization data, where Level 1 plasmids (carbenicillin) are used. The standard error of the growth and GFP rates also increases significantly by utilizing the stronger SJM901, which is not apparent from the original Level 1 promoter characterization. Interestingly, by using the J23100 promoter this error decreases, suggesting that the SJM901 promoter causes more stress to the cells in Level 2 plasmids. We utilized both promoters, since for cell-free measurements, only the stronger SJM901 promoter was sufficient to drive production of mCherry.

With the SJM901 promoter in vivo, the strongest terminators were L3S2P21 (0.37-fold mCherry rate), L3S1P51 (0.73-fold), L3S1P32 (1.26-fold) and L3S1P11 (1.98-fold), each showing a low rate of mCherry fluorescence in comparison to the strong BBa_B0015 terminator. The remaining library members show a 3 to 10 -fold increased mCherry fluorescence rate. The signal intensity of GFP varied between the constructs but remained within a 2.5-fold range of the BBa_B0015 control plasmid. Interestingly, for all of the strong terminators observed in vivo, these also behaved similarly in vitro (Figure 2D). However, of the weaker terminators only L2U5H1 (2.88) and BBa_B0012 (3.09) showed a clear increase in relative mCherry signal. We did observe a broad range of activities, suggesting that small changes in the terminator structure can have significant effects on either GFP or mCherry production. Mechanisms outlined by Chen et al. ${ }^{16}$ can include changes in mRNA degradation, sequestering of the mCherry RBS site through complex secondary structure or decreased translation coupling. Through continued study and a broader in depth characterization of DNA parts for EcoFlex, the changes we have observed could be minimized by implementing the method of Mutalik et al. ${ }^{18}$ or introducing neutral insulator DNA sequences ${ }^{17-19}$ to minimize complex mRNA secondary structure.

ORFs can be fused to a $\mathrm{N}$-terminal fusion tag such as Strep(II) or $\mathrm{His}_{6}$-tag for recombinant protein production and purification. In addition, a combined T7 promoter, PET RBS, $\mathrm{His}_{6}$-tag and thrombin cleavage site can be fused to an ORF of interest, terminator and "destination vector" (e.g., pTU1-ARFP) as a four-part assembly and transformed directly into a lysogenic DE3 strain such as BL21 Gold or KRX for IPTGinducible T7-expression. Alternatively, lower strength constitutive promoters can also be used to lower protein levels to prevent aggregation and inclusion body formation, a problem often encountered with T7-based expression. ${ }^{28}$ In summary, users can construct TUs rapidly with the desired combinations of promoter strength, RBS variants, N-terminal linkers, ORFs and terminator strength and conveniently identify correctly assembled vectors using blue/white (LacZ) or red/white (RFP) screening. Furthermore, for protein production, an
ORF can be assembled with EcoFlex, expressed and purified within $48 \mathrm{~h}$.

As an example, we subcloned CFP and mCherry into the $\mathrm{pET}-15 \mathrm{~b}$ vector using the NdeI and BamHI restriction sites. As a comparison, the same genes were MoClo assembled into pTU1-A with the combined T7 promoter, $\mathrm{His}_{6}$-tag and thrombin cleavage site, together with the BBa_B0015 terminator. We then compared protein production and purification, using BL21 (DE3) Gold pLysS as a host strain. In summary, in comparison to the $\mathrm{pET}-15 \mathrm{~b}$ expression system, our plasmid system produces equivalent levels of the recombinant $\mathrm{His}_{6}$-tagged CFP and mCherry proteins, with a summary of the SDS-PAGE purification of CFP and mCherry shown in Figure 3. To further demonstrate this system, we also

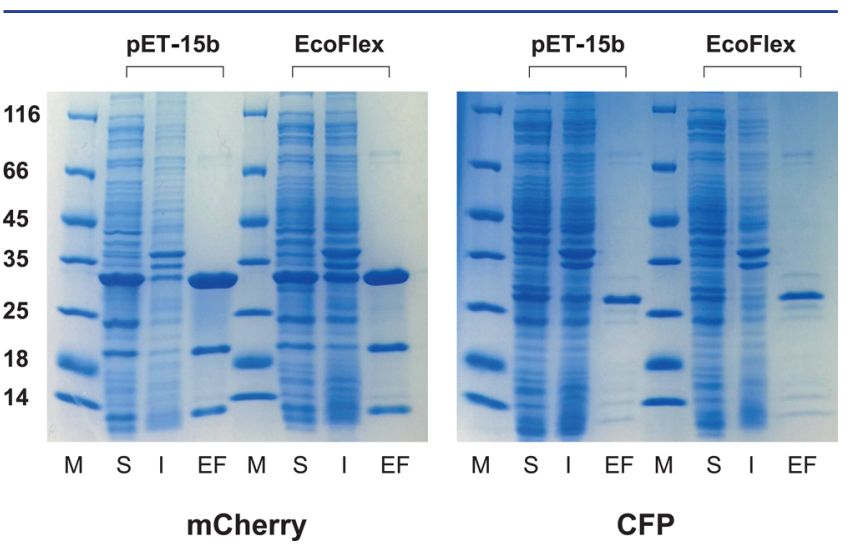

Figure 3. T7 protein production and $\mathrm{His}_{6}$-tag purification of mCherry and CFP. E. coli BL21 Gold (DE3) plasmids strains were grown at 37 ${ }^{\circ} \mathrm{C}$ and induced with $0.4 \mathrm{mM}$ IPTG overnight at $19^{\circ} \mathrm{C}$. Recombinant proteins were purified using IMAC gravity-flow resin and washed with increasing concentrations of imidazole. Figure abbreviations are as follows: M, protein marker; S, soluble extract; I, insoluble proteins; and $\mathrm{EF}$, elution fraction.

tested a variety of proteins (from our own library collection) with this system showing high levels of production in the E. coli KRX autoinduction strain (Figure S5, Table S4).

The assembly of random DNA part libraries for promoter, RBS, ORF and terminator variants is a powerful technique to explore the design space for constructing pathway combinations. Using pooled libraries of promoters and RBS variants for GFP and mCherry in pTU1-A and pTU1-B, respectively, these two TUs were randomly assembled into pTU2-a. Three separate libraries for both GFP and mCherry, with either $3^{2}$, $5^{2}$ or $10^{2}$ variants (for list of parts see Table S4) for coupled promoter and RBS combinations, were built in Level 1 plasmids, creating pTU1-A-GFP and pTU1-B-mCherry. These pooled libraries were sequenced and combinations of pTU1-A-GFP and pTU1-B-mCherry were assembled into pTU2-a, therefore creating theoretical library sizes of $81\left(3^{2}\right.$ variants of GFP combined with $3^{2}$ variants of mCherry), 625 $\left(5^{2}\right.$ variants of GFP combined with $5^{2}$ variants of mCherry) or $10000\left(10^{2}\right.$ variants of GFP combined with $10^{2}$ variants of mCherry). The libraries were analyzed by flow cytometry to determine the relative distribution of pathway variants expressing GFP and mCherry. The fluorescence of GFP and mCherry Level 1 (Figure S7) and Level 2 libraries (Figure 4) was compared to individual Level 1 reference standards (Figure S7) prepared with the J23100, J23108 and J23114 promoters, strong PET RBS and BBa_B0015 terminator, as well as an 

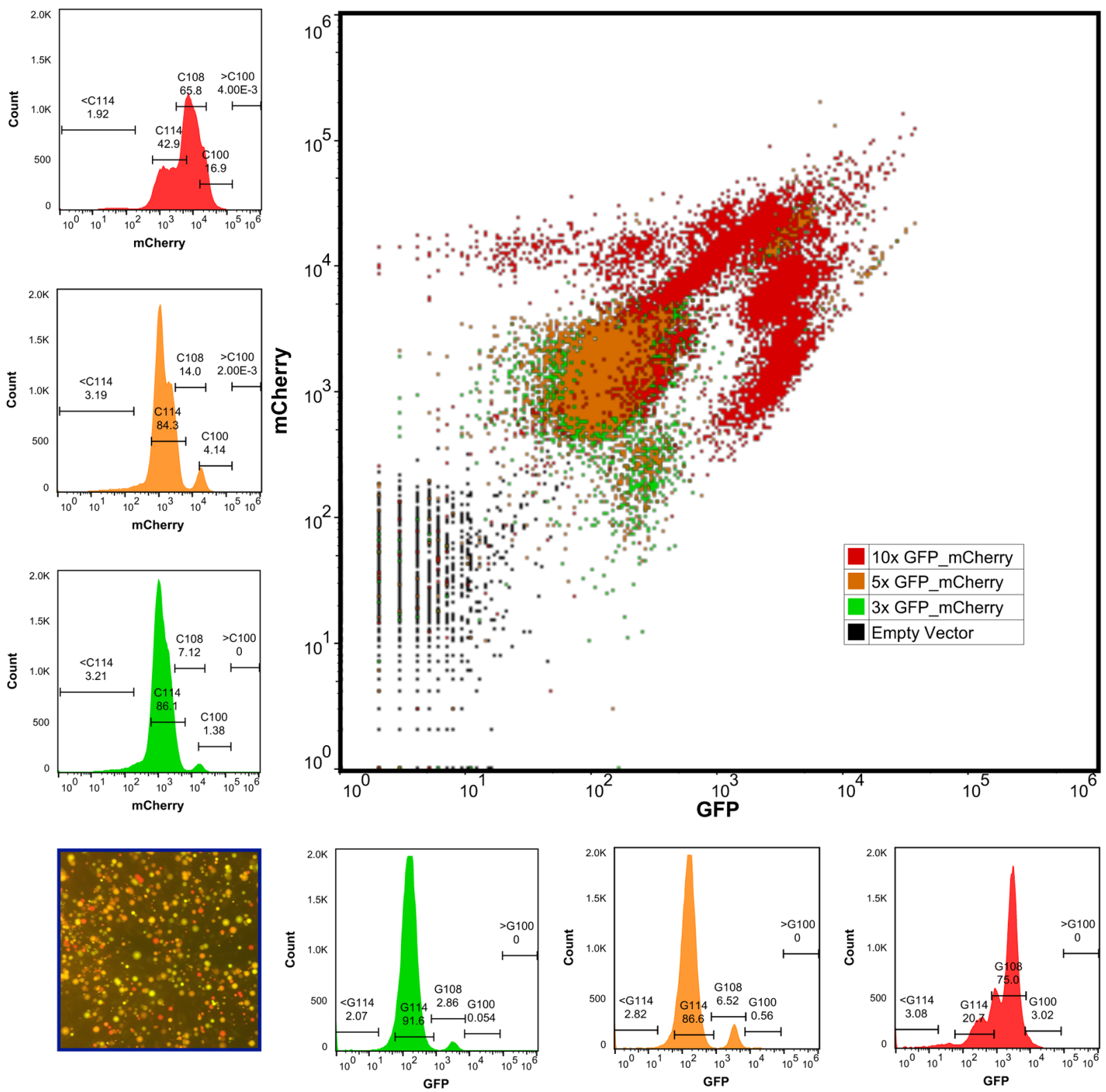

Figure 4. Flow cytometry of GFP and mCherry pooled promoter and RBS libraries. Mixed promoter, RBS libraries were assembled with GFP and mCherry into pTU1-A and pTU1-B, respectively. Instead of picking single colonies, plasmid DNA was prepared directly from liquid culture, checked for purity by restriction digest and sequencing. Level $1 \mathrm{GFP}$ and mCherry libraries were then assembled into pTU2-a and transformed into DH10 $\beta$. Library cultures of Level 1 (Figure S7) and Level 2 were analyzed by flow-cytometry using J23100, J23108 and J23114 promoter standards of GFP (labeled as G100, G108 and G114) and mCherry (labeled as C100, C108 and C114) as a reference of relative strength to estimate population distribution in both $3^{2}$ (9 variants), $5^{2}$ (25 variants) and $10^{2}$ (100 variants) promoter-RBS combinations. These analyses are representative of $\sim 48000$ cells per sample and these data were analyzed using FlowJo (v10.1r5) software.

empty vector control (pTU1-A-lacZ $\alpha$ ). The population distributions of the Level 1 and 2 libraries shifted in response to the diversity of the libraries (Table S5) assembled in the MoClo reactions (Table S6). In general, an increase in the diversity of the library from $3^{2}$, to $5^{2}$ or $10^{2}$, resulted in an increase in diversity for populations that were compared to the expression profiles of the medium (J23108) and strong expression (J23100) profiles of the GFP or mCherry standards. For instance, the population distributions of Level 1 GFP libraries shifted toward an increase in the strong expression profile (J23100-GFP) from 4.41\% $\left(3^{2}\right)$ toward $12.3 \%\left(5^{2}\right)$ and $11 \%\left(10^{2}\right)$ and a marked decrease in the weak expression profile (J23114-GFP) from $70.9 \%\left(3^{2}\right)$ decreasing to $64.1 \%$ $\left(5^{2}\right)$ and $46.3 \%\left(10^{2}\right)$. Additionally, the Level $1 \mathrm{mCherry}$ library profiles displayed an increased representation of the medium (J23108-mCherry) expression from 35.7\% $\left(3^{2}\right)$ toward $45.1 \%$ $\left(5^{2}\right)$ and $57.2 \%\left(10^{2}\right)$, as the diversity of the library was increased.

Analysis of the Level 2 libraries has also shown that as the library diversity was increased from $5^{2}$ to $10^{2}$ there was greater representation of populations in the strong expression profiles of both J23100-GFP ( $5^{2}: 0.68 \%$ to $\left.10^{2}: 4.59 \%\right)$ and J23100mCherry $\left(5^{2}: 4.14 \%\right.$ to $\left.10^{2}: 16.9 \%\right)$. The bias toward lowexpression in the smaller libraries is likely to occur during the overnight growth phase during Level 1 MoClo assembly. E. coli transformed with pooled libraries are likely to be subject to selection pressures in which those cells transformed with weaker promoter and/or RBS combinations were less 

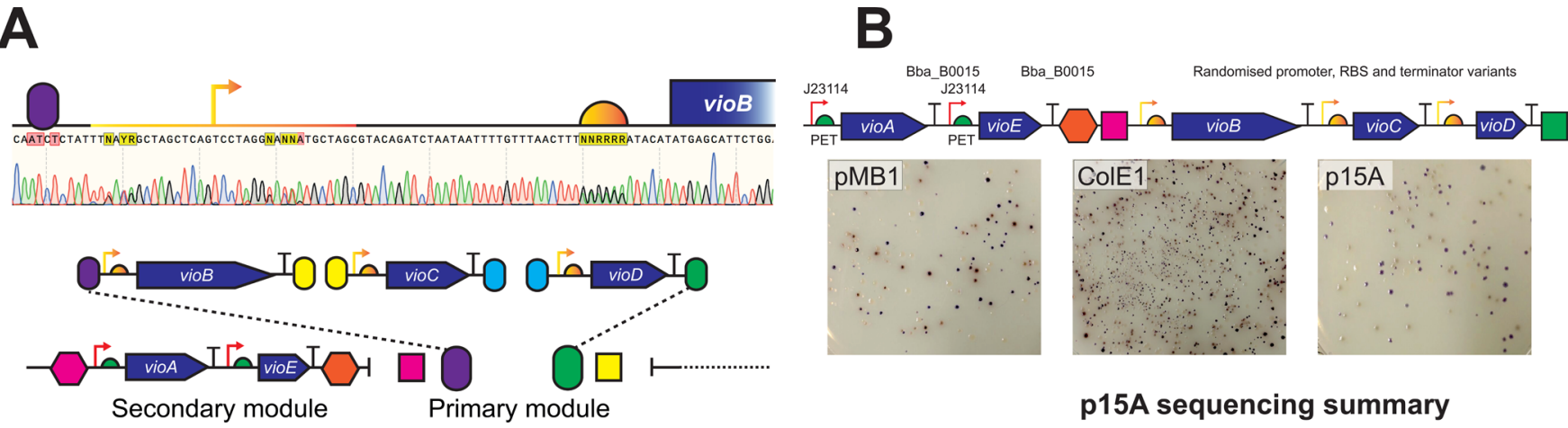

C

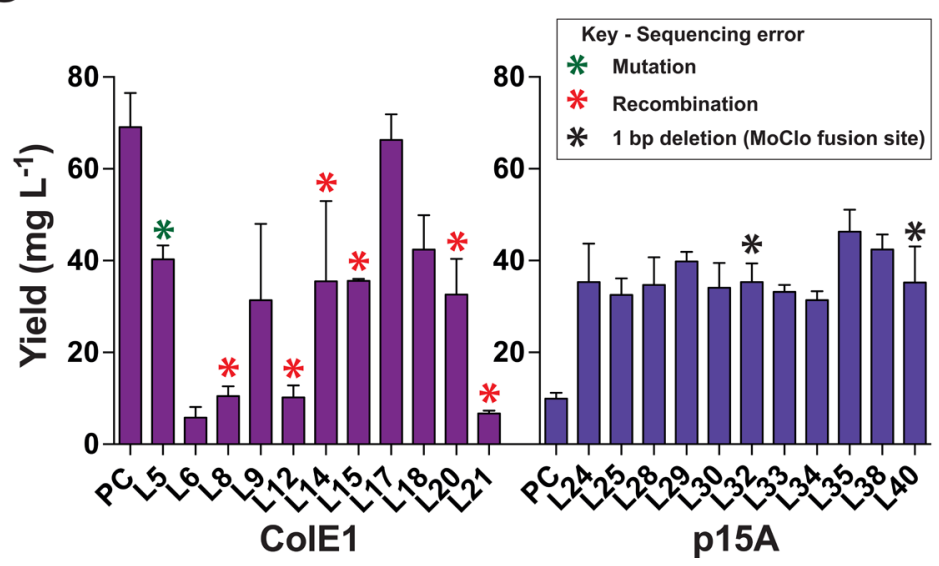

\begin{tabular}{|c|c|c|c|c|c|c|c|c|c|}
\hline \multirow{2}{*}{ ID } & \multicolumn{3}{|c|}{ vioB } & \multicolumn{3}{|c|}{ vioc } & \multicolumn{3}{|c|}{ vioD } \\
\hline & $\Gamma$ & $\cap$ & $\mathrm{T}$ & $\Gamma$ & $\Omega$ & $\mathrm{T}$ & $\Gamma$ & $\cap$ & $\mathrm{T}$ \\
\hline L24 & 902 & 8 & L3S2P21 & 912 & 9 & L3S $2 \mathrm{P} 21$ & 902 & 8 & B0015 \\
\hline L25 & 108 & 7 & L3S2P21 & 912 & 9 & L2U5H08 & 114 & 3 & L2U5HO\& \\
\hline L28 & 114 & 3 & L2U5H08 & 114 & 1 & L2 $2 \mathrm{UH}^{\mathrm{H}} \mathrm{8}$ & 905 & 9 & $\mathrm{~L} 2 \mathrm{U} 5 \mathrm{HOS}$ \\
\hline L29 & 908 & 6 & L3S2P21 & 906 & 4 & L3S2P21 & 908 & 10 & $\mathrm{~L} 2 \mathrm{U} 5 \mathrm{HO}$ \\
\hline L30 & 114 & 10 & L2S2P21 & 901 & 10 & L2 2 5H 08 & 902 & 10 & L2S2P21 \\
\hline L32 & 906 & 6 & B0015 & 906 & $4^{*}$ & L3S2P21 & 108 & 6 & $?$ \\
\hline L33 & 906 & 10 & L2U5H08 & 901 & 9 & L2U5H08 & 908 & 1 & L3S2P21 \\
\hline L34 & 114 & 6 & L2U2H09 & 114 & 4 & L2U5H08 & 114 & 2 & L3S2P21 \\
\hline L35 & 902 & 6 & L3S2P21 & 906 & 7 & L2U5H08 & 915 & 9 & L3S2P21 \\
\hline L38 & 915 & 1 & L3S2P21 & 114 & 4 & L2U2H09 & 902 & 10 & L3S2P21 \\
\hline L40 & 114 & 3 & L3S2P21 & 114 & 4 & L2U2H09 & $905^{*}$ & 8 & L2U5H08 \\
\hline \multicolumn{5}{|c|}{ Promoter/RBS Strength } & \multicolumn{5}{|c|}{ Terminator Strength } \\
\hline & & \multicolumn{3}{|c|}{ Weak } & & & \multicolumn{3}{|c|}{ Weak } \\
\hline & & \multicolumn{3}{|c|}{ Medium } & & & \multicolumn{3}{|c|}{ Medium } \\
\hline & & \multicolumn{3}{|c|}{ Strong } & & & \multicolumn{3}{|c|}{ Strong } \\
\hline
\end{tabular}

Figure 5. Secondary module optimization: Violacein pathway assembly. (A) The vioA and vioE genes were assembled with the J23114 promoter, PET RBS and BBa B0015 terminator in pTU1-A and pTU1-B, respectively and then joined together in pTU2-a. The vioA-vioE fragment was subcloned by BsaI into BpiI digested pTU2S-b to form a vioA-vioE secondary module. Level 1 promoter-RBS-terminator libraries of vioB (pTU1-A), vio C (pTU1-B) and vioD (pTU1-C) were then assembled. Inset sequencing trace demonstrates purity of the library. Library mixtures (pMB1, ColE1 and p15A backbones) were assembled into pTU2S-vioA-vioE-b to complete the pathway. (B) $5 \mu \mathrm{L}$ of assembly mixture was then transformed into 50 $\mu \mathrm{L}$ JM109 competent cells then grown overnight at $37^{\circ} \mathrm{C}$ on $2 \mathrm{YT}$ and chloramphenicol plates. A range of white, brown (PDV, prodeoxyviolacein), light purple and dark purple (violacein/deoxyviolacein) colored colonies were obtained (Table 1). Some of the white colonies developed a pale purple shade after a further day of growth at $30^{\circ} \mathrm{C}$. (C) A selection ColE1 and p15A variants (in as a triplicate biological repeat) were grown in 5 $\mathrm{mL}$ of 2YT liquid culture for high-throughput Bugs2Bases sequencing (Source Bioscience). A number of promoter-RBS regions with insertions, deletions or mutations are highlighted with an asterisk*. Crude violacein content was estimated by UV-Visible spectroscopy as described. ${ }^{39}$ Positive control (PC) with all violacein genes under J23114 promoter, PET RBS and B0015 terminator. (D) Summary of sequencing results for p15A variants showing a mixture of promoter-RBS and terminator combinations. For classification, promoter, RBS and terminator are color coded as strong, medium and weak based on GFP and mCherry characterization data. L32 vioD terminator could not be identified, highlighted as a question mark. Asterisk* indicates single base-pair deletions in p15A library. For full sequencing results, please refer to Supporting Information. Sequencing information is representative of a single culture after additional growth step performed at Source Bioscience.

burdened, in terms of their usage of cellular resources directed to GFP and/or mCherry production, and thus grew more quickly than those cells producing higher levels of the reporter proteins. $^{29}$ As a result, the cells that had lower rates of fluorescent reporter production were overrepresented in the final population. We show that as the size of the library is increased there is likely to be more cells transformed with a larger diversity of medium-to-strong promoter and RBS strength combinations. Therefore, there was a greater diversity of cells with different levels of cellular burden and thus a greater representation of $E$. coli producing higher levels of fluorescent reporter proteins in the final population. While these selection biases exist, this type of approach can provide a quality control check of the efficiency for EcoFlex combinatorial assembly, using fluorescent reporter proteins to rationally enrich library design.

Violacein is a violet pigment first isolated from Chromobacterium violaceum, and the pathway belongs to the indolocarbazole biosynthetic family that utilizes L-tryptophan as a common precursor. ${ }^{30}$ We chose the violacein pathway as a model pathway for EcoFlex combinatorial optimization with the secondary module, as it requires five enzymes (VioA, - B, -C, $-\mathrm{D}$ and $-\mathrm{E}$ ) encoded by the $\mathrm{C}$. violaceum vioABCDE operon, with gene sizes ranging from 576 to $2997 \mathrm{bp}$ and a total size of the synthetic operon of $7,242 \mathrm{bp}$. Herein, we utilize the violacein pathway for optimization as the violet color produced is a qualitative indication of pathway flux during library assembly. $^{31}$

A major consideration for optimizing combinatorial pathways is library size and the limitations of the screening technique. Putting this into perspective, depending on the assembly level and the number of parts included, Golden Gate assemblies typically yield between $10^{2}$ to $10^{5} \mathrm{CFU}$ per transformation. Also, unlike the GFP and mCherry libraries described earlier, a significant decrease in transformation efficiency (approximately 100 -fold) is observed with violacein pathway assembly. For example, during a five TU Level 2 assembly of the complete vio $A B E C D$ pathway with the low-strength J23114 promoter, 
only 20-30 purple colonies were obtained from either JM109 or KRX high efficiency $\left(1 \times 10^{8}\right.$ CFU per $\mu$ g DNA $)$ competent cells. Toxicity was also noted during optimization of violacein with VEGAS cloning in Brewer's yeast. ${ }^{13}$ To tackle this problem and provide an aid to debug pathway diversity toward a more favorable design space, we decided to introduce a secondary module site into the EcoFlex cloning scheme (Figure 1C). This secondary module encodes two BpiI sites (see SI text), which provide compatible overhangs ( $5^{\prime}$ CTAT) and ( $3^{\prime}$ GTAC) for transferring a subpathway (or other DNA parts) from the Level 2 plasmids pTU2-A, -a, or -b through BsaI digest, thus allowing entry of 2-5 TUs into the secondary site.

As an example, a constant subpathway for the vioA and vioE genes was assembled into the RFP-containing pTU1-A and -B destination plasmids respectively, with a J23114 promoter, PET RBS and BBa_B0015 terminator. Next, the vioA and vioE TUs were assembled into pTU2-a, before subcloning of this module by BsaI digest into the BpiI-linearized secondary module plasmid pTU2S-b, creating pTU2S-vioA-vioE-b with either pMB1 (high-copy), ColE1 (medium-copy) and p15A (lowcopy) origins of replication. Next, pooled promoter, RBS and terminator libraries, and $v i o B, v i o C$ and $v i o D$ genes were assembled in pTU1-A-lacZ $\alpha$, pTU1-B-lacZ $\alpha$ and pTU1-Clac $Z \alpha$ respectively. $50 \mu \mathrm{L}$ of the transformation mixture was plated onto carbenicillin agar to estimate Level 1 efficiency using blue/white colony screening ( $>99 \%$ efficiency), while the remaining mixture $(\sim 200 \mu \mathrm{L})$ was directly inoculated into 10 $\mathrm{mL}$ of LB medium (with carbenicillin) and grown overnight at $30{ }^{\circ} \mathrm{C}$. The library plasmid DNA was isolated, verified for homogeneity by restriction digest and sequenced (Figure 5A). Level 1 promoter-RBS-terminator libraries of vioB, vioC and vioD were then assembled into pTU2S-vioA-vioE-b (pMB1, ColE1 and p15A) using BsmBI and T4 DNA ligase. Five $\mu \mathrm{L}$ of the reaction mixture was then transformed into $50 \mu \mathrm{L}$ of $E$. coli JM109 competent cells and plated onto chloramphenicol plates and counted for CFUs (Figure 5B and Table 1). We utilized

Table 1. CFUs for Violacein Pathway Optimization with the Secondary Module

\begin{tabular}{lcccc}
\multicolumn{5}{c}{ CFU phenotypes } \\
\hline origin of replication & violacein (high) & violacein (low) & PDV & white \\
pMB1 (high) & 27 & 7 & 18 & 48 \\
ColE1 (medium) & 88 & 6 & 57 & 115 \\
p15A (low) & 270 & 0 & 118 & 154 \\
\hline
\end{tabular}

JM109 instead of DH10 $\beta$ as we have observed instability issues with complex biosynthetic pathways with DH10 $\beta$. For example, transformation of the lycopene or violacein pathway (pTU2-Avio $A B C D E$ - positive pathway control) yields a low percentage of white colonies, which upon plasmid purification yields the same mini-prep restriction digest map as the original plasmid. Whilst we did not pursue sequencing to identify potential mutations, it is known that $\mathrm{DH} 10 \beta$ has a high basal mutation frequency. ${ }^{32}$ With JM109 transformants, we observed a variety of CFU phenotypes indicating production of violacein intermediates (Table 1). Unlike the low-efficiency 5 TU vio $A B C D E$ assembly, approximately $10^{2}-10^{3}$ CFUs were obtained with the plasmids containing the pMB1, ColE1 or p15A origins of replication (Table 1).

Initially, we assayed violacein production and sequenced the library parts around each TU (vioB, vioC and vioD) in a few colonies from the library transformation plate with $\mathrm{pMB} 1$ origin, and found that most of them contain a mutated promoter or RBS in at least one of the vioB, vioC or vioD TUs after overnight growth (see SI text and Figure S9-10). It is also interesting to note that some of the clones after overnight liquid or plate growth lose their ability to produce violacein, which is consistent with a mutation or recombination event. To test whether this was linked to plasmid copy number, we also screened colonies from the library transformation using ColE1 (medium-copy) and p15A (low-copy) origins of replication.

Focusing on the ColE1 and p15A plasmid variants, we restreaked purple colonies onto fresh plates and single colonies were then grown in biological triplicate to semi-quantify crude violacein, while liquid culture aliquots were sent for highthroughput 96-well sequencing (Bugs2Bases, Source Bioscience) of the vioB, vioC and vioD library regions, which requires an additional subculturing step before DNA purification and sequencing. For ColE1 plasmid variants, only $5 / 11$ clones were absent of mutations, while for p15A clones, this increased to $9 / 11$ clones. However, in the two p15A clones that did contain a mutation (L32 vioC RBS and L40 vioD promoter), these were single base-pair deletions immediately adjacent to the MoClo fusion site and could be derived from initial Level 1 assembly (Figure S13). In addition, the sequencing trace of the $\mathrm{L} 32$ vioD terminator was weak, despite repeat sequencing. The impact of the violacein pathway as a strong negative selection marker also appeared to amplify the frequency of these mutation events. Golden Gate and MoClo assembly is renowned to be highly efficient and a restriction digest of DNA is generally considered acceptable for qualitycontrol checking. ${ }^{33}$ In contrast, the mutations found in pMB1 and ColE1 variants generally represent deletions (probable recombination events), which form spontaneously during subculturing. We speculate that these events are due to repetitive promoter, RBS and terminator sequences ${ }^{16,34}$ used during assembly, and strong selection pressures caused by violacein production. This is further supported by the fact that no mutations or deletions were detected within the sequenced regions of the vioB, vioC and vioD ORFs, thus mutations only seemed to occur within the promoter, RBS or terminator sequences. This provides a strong focal point for improvement in our future studies of library design. Please see Supporting Information for a full summary of these findings.

In general, the library colonies gave a wide range of crude violacein yields, with the highest producing strain L15 giving $66.3 \pm 5.6 \mathrm{mg} \mathrm{L}^{-1}$ and the lowest strain L21 giving $3.6 \pm 1.7$ $\mathrm{mg} \mathrm{L}^{-1}$ (Figure 5D). However, the ColE1 positive control strain (with all violacein genes under J23114 promoter, PET RBS and BBa_B0015 terminator) had the highest crude violacein yield at $69.1 \pm 7.4 \mathrm{mg} \mathrm{L}^{-1}$, whereas the equivalent p15A positive control strain produced $9.9 \pm 1.3 \mathrm{mg} \mathrm{L}^{-1}$, the lowest among the p15A library strains (Figure 5). From the sequencing information, the ColE1 and p15A libraries were predominated by weak-medium strength promoters and a range of RBS combinations. Interestingly, the strongest promoter library member SJM901 appears in two instances with vioC, but it was only found in the p15A variants. It should also be noted that when violacein production was previously optimized with ePathOptimize using five engineered variants of the T7 promoter, all strong producing clones (up to $60-240 \mathrm{mg} \mathrm{L}^{-1}$ crude violacein) were predominated by combinations of the weakest T7 promoter variant. ${ }^{35}$ Although our study only used constitutive promoters, the frequent occurrence of weak promoters with strong pathway variants is a shared common 
theme between these two studies. Fine-tuning of violacein flux was also recently examined by rationally minimizing library size of RBS sites in a recent study. ${ }^{36}$

For the terminators, there was a strong bias toward the L3S2P21, L2U5H08, L2U2H08 and Bba B0015 members with only a single occurrence of Bba_B0012. Considering all variants of the EcoFlex library were included at approximately the same concentration at the initial Level 1 assembly, it is still unclear why certain terminators become more frequent at the final stage of selection of violacein CFUs.

While the EcoFlex kit is primarily designed for E. coli, we are also interested in expanding this toolkit toward a universal cloning system between alternative prokaryotic hosts that offer attractive advantages in biotechnology, such as thermophilic growth, utilization of alternative carbon feedstocks, resistance to growth-inhibitory metabolites and high titer natural product synthesis. For example, a pathway design could be studied in a non-traditional host and an individual gene from the same pathway could then be rapidly swapped into E. coli for enzyme or structural studies. Furthermore, while we demonstrate that the secondary module can be used for the optimization of biosynthetic pathways, this feature could also be used for constructing novel shuttle vectors or genome engineering tools. EcoFlex provides a foundation for the design and implementation of a universal cloning system as we look toward arcane chassis, ${ }^{37}$ which are becoming increasingly important for future synthetic biology directions. In summary, the EcoFlex kit provides a simplified cloning scheme that can be used for a variety of synthetic biology applications for E. coli and, in the future, alternative prokaryotic host systems.

\section{METHODS}

Bacterial Strains and Growth. Routine bacterial growth was performed at $37{ }^{\circ} \mathrm{C}$ in LB broth, with agar $\left(15 \mathrm{~g} \mathrm{~L}^{-1}\right)$, carbenicillin $\left(100 \mu \mathrm{g} \mathrm{mL}^{-1}\right)$, kanamycin $\left(50 \mu \mathrm{g} \mathrm{mL}^{-1}\right)$ or chloramphenicol (35 $\left.\mu \mathrm{g} \mathrm{mL}^{-1}\right)$ as needed. Chemically competent E. coli DH10 $\beta$ (NEB), JM109 (Promega) and KRX (Promega) were used for routine transformations and maintenance of plasmids.

Molecular Biology. Restriction enzymes (NEB), T4 polynucleotide kinase (NEB), T4 DNA ligase (Promega), Phusion (Agilent) and Q5 DNA polymerase (NEB) were used for routine cloning methods. Molecular biology kits were purchased from Qiagen (QIAprep Miniprep kit and QIAquick gel extraction kit). Primers and the lacZ $\alpha$ fragment (see SI text) were synthesized by IDT. DNA parts and primer sequences are provided in the Supporting Information. Routine and highthroughput Bugs2Bases DNA sequencing was performed by Source Bioscience, Cambridge.

EcoFlex Assembly Conditions. For Level 1 and 3 assembly, $100 \mathrm{ng}$ of each DNA part was combined with 50 ng of destination plasmid and incubated with BsaI and T4 ligase for $15-30$ cycles of $5 \mathrm{~min}$ at $37^{\circ} \mathrm{C}$ and $10 \mathrm{~min}$ at $16^{\circ} \mathrm{C}$, followed by $5 \mathrm{~min}$ at $50{ }^{\circ} \mathrm{C}$ and $5 \mathrm{~min}$ at $80^{\circ} \mathrm{C}$. For Level 2 assembly, BsmBI was used as the type IIS restriction enzyme and the reaction was incubated at $37{ }^{\circ} \mathrm{C}$ overnight. Five $\mu \mathrm{L}$ of Golden Gate reaction mix was transformed into $50 \mu \mathrm{L}$ of chemically competent E. coli DH10 $\beta$, JM109 or KRX cells using heat shock transformation. After recovery in $200 \mu \mathrm{L}$ of SOC medium (NEB), 50-100 $\mu \mathrm{L}$ of cells were plated onto $\mathrm{LB}$ antibiotic plates and grown at $37^{\circ} \mathrm{C}$ overnight. For assembly of Level 1 libraries, a pooled library of promoters or RBS (100 ng $\mathrm{uL}^{-1}$ ) variants were assembled in a Level 1 assembly reaction and transformed into $\mathrm{DH} 10 \beta$. Instead of incubating on plates, $200 \mu \mathrm{L}$ of the SOC mixture was directly inoculated into $10 \mathrm{~mL}$ of $2 \mathrm{YT}$ medium with antibiotic and grown overnight at $30{ }^{\circ} \mathrm{C}$ and $160 \mathrm{rpm}$ shaking. High purity library mini-preps was verified by restriction digest and sequencing.

Flow Cytometry. Golden gate combinatorial library reactions were transformed into $E$. coli and grown overnight in $1 \mathrm{~mL} 2 \mathrm{YT}$ and antibiotics at $30{ }^{\circ} \mathrm{C}$. Cell populations were diluted into $1 \mathrm{X}$ phosphate buffered saline $(2: 1000)$ and loaded for detection into a S3e Cell sorter (Biorad, CA, USA). GFP detection (FL1, Excitation $488 \mathrm{~nm}$, Emission 525/30 nm) and mCherry detection (FL3, Excitation $561 \mathrm{~nm}$, Emission 615/25 $\mathrm{nm}$ ) for approximately 48000 cells per sample, was carried out and analyzed using FlowJo (v10.1r5) software (FlowJo, LLC, Oregon, USA). In order to analyze the library populations, a gating strategy was used in which reference regions were quantified. To do this, Level 1 plasmids were prepared with either GFP or mCherry assembled with low (J23114), lowmedium (J23108) and high (J23100) promoter strengths, the PET RBS and BBa_B0015 terminator. Single plasmid clones were transformed into $\mathrm{DH} 10 \boldsymbol{\beta}$. The distribution of library populations was then estimated by overlapping with the known reference standards.

Cell-Free Protein Synthesis. On the basis of the protocol from Sun et al., ${ }^{38}$ a cell-extract was prepared from E. coli Rosetta (DE3) pLysS and reactions were monitored in a CLARIOStar (BMG, Germany) plate reader. For full details, please see SI text and Table S2.

In Vivo Growth and Fluorescence Measurements. Promoter, RBS and terminator parts were characterized in E. coli $\mathrm{DH} 10 \beta$. Single colonies were picked in triplicate and grown overnight at $30{ }^{\circ} \mathrm{C}$ in a 24 -well plate in $2 \mathrm{~mL}$ of $2 \mathrm{YT}$ medium with antibiotics. Cell density was measured in a CLARIOStar plate reader at $600 \mathrm{~nm}$ and cells were subcultured into $100 \mu \mathrm{L}$ of $2 \mathrm{YT}$ with antibiotics in a 96-well Greiner plate to a starting $\mathrm{OD}_{600}$ of 0.05 . Plates were sealed with a BreatheEasy membrane (Sigma) and grown at $30^{\circ} \mathrm{C}$ for $6-12 \mathrm{~h}$ at 600 $\mathrm{rpm}$. $\mathrm{OD}_{600}$, GFP and mCherry measurements were recorded every $10 \mathrm{~min}$ (see SI text). Data analysis of characterization data was quantified as described previously with one modification. ${ }^{27}$ Rates of GFP and mCherry production were quantified during exponential phase from an average of triplicate data over a $1 \mathrm{~h}$ time period. For cell-free activity measurements only, mCherry fluorescence accumulates significantly later than GFP and was measured at a different time point.

Protein Production and Purification. Plasmids (pET15b or EcoFlex) were transformed into BL21 Gold (DE3) pLysS. A $10 \mathrm{~mL} 2 \mathrm{YT}$ overnight culture with antibiotics was grown overnight and $0.5 \mathrm{~mL}$ was subcultured into $100 \mathrm{~mL} 2 \mathrm{YT}$ and grown at $37{ }^{\circ} \mathrm{C}, 200 \mathrm{rpm}$ until an $\mathrm{OD}_{600}$ of 1.0 was reached. IPTG $(0.4 \mathrm{mM})$ was added and the culture was left for $16 \mathrm{~h}$ at $19{ }^{\circ} \mathrm{C}, 200 \mathrm{rpm}$. Gravity flow immobilized metal ion chromatography (IMAC) purification was performed. Please see SI text for further details.

\section{ASSOCIATED CONTENT}

\section{Supporting Information}

The Supporting Information is available free of charge on the ACS Publications website at DOI: 10.1021/acssynbio.6b00031.

Table S1-S14 and Figures S1-S13, as well as oligonucleotide and DNA part sequences. (PDF) 
DNA part characterization and sequencing, 1. (XLSX)

DNA part characterization and sequencing, 2. (XLSX)

DNA part characterization and sequencing, 3. (XLSX)

\section{AUTHOR INFORMATION}

\section{Corresponding Author}

*Tel: +44 (0) 2075945327. Fax: +44 (0) 207594 30. E-mail: p. freemont@imperial.ac.uk.

\section{Author Contributions}

S.M., K.P. and P.F. designed the study, analyzed data, and wrote the manuscript. S.M. and H.L. assembled EcoFlex. S.M. H.L., R.K., S.C. and D.B. performed the experiments.

\section{Notes}

The authors declare no competing financial interest.

\section{ACKNOWLEDGMENTS}

SM and RK are postdoctoral researchers funded by the EPSRC grants EP/K038648/1 (Frontier Engineering) and EP/ J02175X/1 (Flowers Consortium), respectively. HL is funded by a studentship award from Imperial College London. We would also like to thank Dr Lisa Goers and Dr James Arpino from Imperial College London for providing plasmids for cloning.

\section{REFERENCES}

(1) Ran, N., Zhao, L., Chen, Z., and Tao, J. (2008) Recent applications of biocatalysis in developing green chemistry for chemical synthesis at the industrial scale. Green Chem. 10, 361.

(2) Casini, A., Storch, M., Baldwin, G. S., and Ellis, T. (2015) Bricks and blueprints: methods and standards for DNA assembly. Nat. Rev. Mol. Cell Biol. 9, 1-9.

(3) Engler, C., Kandzia, R, and Marillonnet, S. (2008) A one pot, one step, precision cloning method with high throughput capability. PLoS One 3, e3647.

(4) Engler, C., and Marillonnet, S. (2011) Generation of families of construct variants using golden gate shuffling. Methods Mol. Biol. 729, $167-81$.

(5) Engler, C., Gruetzner, R., Kandzia, R., and Marillonnet, S. (2009) Golden gate shuffling: A one-pot DNA shuffling method based on type IIS restriction enzymes. PLoS One 4, e5553.

(6) Weber, E., Gruetzner, R., Werner, S., Engler, C., and Marillonnet, S. (2011) Assembly of designer TAL effectors by Golden Gate cloning. PLoS One 6, e19722.

(7) Sarrion-Perdigones, A., Falconi, E. E., Zandalinas, S. I., Juárez, P., Fernández-del-Carmen, A., Granell, A., and Orzaez, D. (2011) GoldenBraid: An iterative cloning system for standardized assembly of reusable genetic modules. PLoS One 6, e21622.

(8) Werner, S., Engler, C., Weber, E., Gruetzner, R., and Marillonnet, S. (2012) Fast track assembly of multigene constructs using Golden Gate cloning and the MoClo system. Bioeng. Bugs 3, 38-43.

(9) Weber, E., Engler, C., Gruetzner, R., Werner, S., and Marillonnet, S. (2011) A modular cloning system for standardized assembly of multigene constructs. PLoS One 6, e16765.

(10) Engler, C., Youles, M., Gruetzner, R., Ehnert, T.-M., Werner, S., Jones, J. D. G., Patron, N. J., and Marillonnet, S. (2014) A golden gate modular cloning toolbox for plants. ACS Synth. Biol. 3, 839-43.

(11) Sarrion-Perdigones, A., Vazquez-Vilar, M., Palací, J., Castelijns, B., Forment, J., Ziarsolo, P., Blanca, J., Granell, A., and Orzaez, D. (2013) GoldenBraid 2.0: a comprehensive DNA assembly framework for plant synthetic biology. Plant Physiol. 162, 1618-31.

(12) Agmon, N., Mitchell, L. A., Cai, Y., Ikushima, S., Chuang, J., Zheng, A., Choi, W.-J., Martin, J. A., Caravelli, K., Stracquadanio, G., and Boeke, J. D. (2015) Yeast Golden Gate (yGG) for the Efficient Assembly of S. cerevisiae Transcription Units. ACS Synth. Biol. 4, 8539.
(13) Mitchell, L. a., Chuang, J., Agmon, N., Khunsriraksakul, C., Phillips, N. a., Cai, Y., Truong, D. M., Veerakumar, A., Wang, Y., Mayorga, M., Blomquist, P., Sadda, P., Trueheart, J., and Boeke, J. D. (2015) Versatile genetic assembly system (VEGAS) to assemble pathways for expression in S. cerevisiae. Nucleic Acids Res. 43, 66206630.

(14) Kakui, Y., Sunaga, T., Arai, K., Dodgson, J., Carazo-salas, R., Ji, L., Csika, A., and Sato, M. (2015) Module-based construction of plasmids for chromosomal integration of the fission yeast Schizosaccharomyces pombe. Open Biol. 5, 150054.

(15) Iverson, S., Haddock, T. L., Beal, J., and Densmore, D. (2015) CIDAR MoClo: Improved MoClo Assembly Standard and New E. coli Part Library Enables Rapid Combinatorial Design for Synthetic and Traditional Biology. ACS Synth. Biol. 5, 99.

(16) Chen, Y.-J., Liu, P., Nielsen, A. A. K., Brophy, J. A. N., Clancy, K., Peterson, T., and Voigt, C. A. (2013) Characterization of 582 natural and synthetic terminators and quantification of their design constraints. Nat. Methods 10, 659-664.

(17) Casini, A., Christodoulou, G., Freemont, P. S., Baldwin, G. S., Ellis, T., and MacDonald, J. T. (2014) R2oDNA designer: computational design of biologically neutral synthetic DNA sequences. ACS Synth. Biol. 3, 525-8.

(18) Mutalik, V. K., Guimaraes, J. C., Cambray, G., Lam, C., Christoffersen, M. J., Mai, Q.-A., Tran, A. B., Paull, M., Keasling, J. D., Arkin, A. P., and Endy, D. (2013) Precise and reliable gene expression via standard transcription and translation initiation elements. Nat. Methods 10, 354-60.

(19) Lou, C., Stanton, B., Chen, Y.-J., Munsky, B., and Voigt, C. A. (2012) Ribozyme-based insulator parts buffer synthetic circuits from genetic context. Nat. Biotechnol. 30, 1137-42.

(20) Anderson, J. C., Dueber, J. E., Leguia, M., Wu, G. C., Goler, J. A., Arkin, A. P., and Keasling, J. D. (2010) BglBricks: A flexible standard for biological part assembly. J. Biol. Eng. 4, 1.

(21) Beyer, H. M., Gonschorek, P., Samodelov, S. L., Meier, M., Weber, W., and Zurbriggen, M. D. (2015) AQUA Cloning: A Versatile and Simple Enzyme-Free Cloning Approach. PLoS One 10, e0137652.

(22) Storch, M., Casini, A., Mackrow, B., Fleming, T., Trewhitt, H., Ellis, T., and Baldwin, G. S. (2015) BASIC: A New Biopart Assembly Standard for Idempotent Cloning Provides Accurate, Single-Tier DNA Assembly for Synthetic Biology. ACS Synth. Biol. 4, 781-7.

(23) Torella, J. P., Lienert, F., Boehm, C. R., Chen, J.-H., Way, J. C. and Silver, P. A. (2014) Unique nucleotide sequence-guided assembly of repetitive DNA parts for synthetic biology applications. Nat. Protoc. 9, 2075-89.

(24) Gibson, D. G., Young, L., Chuang, R.-Y., Venter, J. C., Hutchison, C. a, and Smith, H. O. (2009) Enzymatic assembly of DNA molecules up to several hundred kilobases. Nat. Methods 6, 343-345.

(25) Li, M. Z., and Elledge, S. J. (2012) SLIC: a method for sequence- and ligation-independent cloning. Methods Mol. Biol. 852, 51-9.

(26) Kelly, J. R., Rubin, A. J., Davis, J. H., Ajo-Franklin, C. M., Cumbers, J., Czar, M. J., de Mora, K., Glieberman, A. L., Monie, D. D., and Endy, D. (2009) Measuring the activity of BioBrick promoters using an in vivo reference standard. J. Biol. Eng. 3, 4.

(27) Chappell, J., Jensen, K., and Freemont, P. S. (2013) Validation of an entirely in vitro approach for rapid prototyping of DNA regulatory elements for synthetic biology. Nucleic Acids Res. 41, 34713481.

(28) Jürgen, B., Breitenstein, A., Urlacher, V., Büttner, K., Lin, H., Hecker, M., Schweder, T., and Neubauer, P. (2010) Quality control of inclusion bodies in Escherichia coli. Microb. Cell Fact. 9, 41.

(29) Ceroni, F., Algar, R., Stan, G.-B., and Ellis, T. (2015) Quantifying cellular capacity identifies gene expression designs with reduced burden. Nat. Methods 12, 415-418.

(30) Knaggs, A. R. (2003) The biosynthesis of shikimate metabolites. Nat. Prod. Rep. 20, 119-36.

(31) Balibar, C. J., and Walsh, C. T. (2006) in vitro biosynthesis of violacein from L-tryptophan by the enzymes VioA-E from Chromobacterium violaceum. Biochemistry 45, 15444-15457. 
(32) Durfee, T., Nelson, R., Baldwin, S., Plunkett, G., Burland, V., Mau, B., Petrosino, J. F., Qin, X., Muzny, D. M., Ayele, M., Gibbs, R. a., Csörgo, B., Pósfai, G., Weinstock, G. M., and Blattner, F. R. (2008) The complete genome sequence of Escherichia coli DH10B: Insights into the biology of a laboratory workhorse. J. Bacteriol. 190, 25972606.

(33) Lee, M. E., DeLoache, W. C., Cervantes, B., and Dueber, J. E. (2015) A Highly Characterized Yeast Toolkit for Modular, Multipart Assembly. ACS Synth. Biol. 4, 975-86.

(34) Sleight, S. C., Bartley, B. A., Lieviant, J. A., and Sauro, H. M. (2010) Designing and engineering evolutionary robust genetic circuits. J. Biol. Eng. 4, 12.

(35) Jones, J. A., Vernacchio, V. R., Lachance, D. M., Lebovich, M., Fu, L., Shirke, A. N., Schultz, V. L., Cress, B., Linhardt, R. J., and Koffas, M. a. G. (2015) ePathOptimize: A Combinatorial Approach for Transcriptional Balancing of Metabolic Pathways. Sci. Rep. 5, 11301.

(36) Jeschek, M., Gerngross, D., and Panke, S. (2016) Rationally reduced libraries for combinatorial pathway optimization minimizing experimental effort. Nat. Commun. 7, 11163.

(37) Eisenstein, M. (2016) Living factories of the future. Nature 531, 401-403.

(38) Sun, Z. Z., Hayes, C. A., Shin, J., Caschera, F., Murray, R. M., and Noireaux, V. (2013) Protocols for implementing an Escherichia coli based TX-TL cell-free expression system for synthetic biology. $J$. Visualized Exp. 50762, e50762.

(39) Durán, N., Justo, G. Z., Ferreira, C. V., Melo, P. S., Cordi, L., and Martins, D. (2007) Violacein: properties and biological activities. Biotechnol. Appl. Biochem. 48, 127-33. 